\title{
Evaluation of Real-Time PCR for Detection of and Discrimination between Bordetella pertussis, Bordetella parapertussis, and Bordetella holmesii for Clinical Diagnosis
}

\author{
Kate E. Templeton, ${ }^{1}$ Sitha A. Scheltinga, ${ }^{1}$ Anneke van der Zee, ${ }^{2}$ Bram M. W. Diederen, ${ }^{1}$ \\ Anna M. Kruijssen, ${ }^{3}$ Herman Goossens, ${ }^{1,4}$ Ed Kuijper, ${ }^{1}$ and Eric C. J. Claas ${ }^{1 *}$ \\ Department of Medical Microbiology, Center of Infectious Diseases, ${ }^{1}$ and Department of Pediatrics, ${ }^{3}$ Leiden University \\ Medical Center, Leiden, and Laboratory of Molecular Microbiology, St. Elisabeth Hospital, Tilburg, ${ }^{2}$ \\ The Netherlands, and Department of Microbiology, University of Antwerp, Antwerp, Belgium ${ }^{4}$ \\ Received 31 January 2003/Returned for modification 29 April 2003/Accepted 24 June 2003
}

\begin{abstract}
PCR is increasingly being used as a diagnostic test for the detection of Bordetella pertussis and Bordetella parapertussis DNA, as it has improved sensitivity and specificity in comparison to conventional techniques. The assay described here uses the two insertion sequences IS481 and IS1001 for B. pertussis and B. parapertussis, respectively, with detection by molecular beacons. The real-time PCR for IS481 detects both $B$. pertussis and Bordetella holmesii, and the real-time PCR for IS1001 detects both B. parapertussis and B. holmesii. By performing both assays discrimination between $B$. pertussis and $B$. parapertussis can be obtained. The sensitivity was 1 to $10 \mathrm{CFU} / \mathrm{ml}$ for $B$. pertussis, $10 \mathrm{CFU} / \mathrm{ml}$ for $B$. parapertussis, and $10 \mathrm{CFU} / \mathrm{ml}$ for $B$. holmesii in both assays. The clinical sensitivity of the $B$. pertussis assay was not affected by duplexing with an internal control PCR. Real-time PCR, conventional PCR, and culture were performed on 57 clinical samples. Eight of the 57 (14\%) were found positive by culture, 19 of $57(33 \%)$ were found positive by conventional PCR, and 22 of 57 (39\%) were found positive by real-time PCR. One sample was inhibitory. When the B. pertussis assay was compared with a clinical standard for $B$. pertussis infection, sensitivity was 38,83 , and $100 \%$ and specificity was 100, 97, and 97\% for culture, conventional PCR, and real-time PCR, respectively. The real-time PCR designed for $B$. pertussis and $B$. parapertussis provides sensitive and specific diagnosis of $B$. pertussis and $B$. parapertussis infections and is therefore suitable for implementation in the diagnostic laboratory.
\end{abstract}

Bordetella pertussis is the causative agent of whooping cough (pertussis), an infectious disease that occurs worldwide with a high prevalence among young unvaccinated infants $(1,27)$ and recently has reemerged in highly vaccinated populations (10). Pertussis-like symptoms are also caused by related species of bacteria, including Bordetella parapertussis and Bordetella holmesii (16), although $B$. parapertussis infections are usually less severe (8).

Laboratory diagnosis is traditionally based on culture, which is regarded as the "gold standard" and is highly specific but whose sensitivity in comparison to PCR or serology varies between 7 and 36\% (13, 29, 31). Furthermore, culturing depends on specimen quality, rapid transportation, and laboratory expertise. This is an important factor in all diagnostic assays requiring propagation of the pathogen, but in the case of $B$. pertussis these requirements may be more acute. Adequate serological detection of $B$. pertussis generally requires paired samples, although immunoglobulin A or immunoglobulin $G$ measurements in a single serum can also be used (2). So far no serologic assay has been approved for diagnostic use in the United States because diagnostic criteria have not been accepted and no method has been validated between laboratories (22).

PCR is increasingly being used as a diagnostic test for the

\footnotetext{
* Corresponding author. Mailing address: Department of Medical Microbiology, Center of Infectious Diseases, Leiden University Medical Center, P.O. Box 9600, 2300 RC Leiden, The Netherlands. Phone: 3171526 3650. Fax: 3171524 8148. E-mail: E.Claas@LUMC.NL.
}

detection of B. pertussis and B. parapertussis DNA (4, 7, 18, 26, 30). In a comparison of different PCR assays used in pertussis vaccine studies, all assays provided an increased sensitivity for detection of pertussis cases by at least $70 \%$ in comparison to culture (23). Various PCR protocols have been developed that target different regions of the genome: insertion sequences IS481 and IS1001 (4, 6, 13, 15, 26, 30), the pertussis toxin promoter region $(6,15,20,26)$, the porin gene $(5)$, and the adenylate cyclase gene (3). The achievements of the PCR assays targeting these different genes have not been widely compared. The insertion sequences have been found to be more sensitive than the pertussis toxin promoter (26), but other workers have shown comparable sensitivities for the insertion sequences and the porin gene (5) and the insertion sequences and the pertussis toxin promoter (25). The copy number of the insertion sequence IS481 in B. pertussis is approximately 100 , the copy number of the insertion sequence IS1001 in B. parapertussis is 20 to 21 (32), and for $B$. holmesii the copy number is unknown. Recently, diagnostic applications of PCR formats have been significantly improved by implementing fluorescent, real-time detection of the amplification products. By adding fluorescent probes to the amplification reaction PCR products can be detected as they are generated. For qualitative PCR applications real-time PCR offers advantages, as post-PCR processing is redundant. This reduces the time to results and in addition the risk of contamination. Some real-time PCR assays have been described for $B$. pertussis $(11,21,26)$. For appropriate application of these PCR assays consensus recommendations have been formulated (17), and so far only one conven- 
TABLE 1. Bacterial species and strains

\begin{tabular}{|c|c|c|}
\hline Species & Strain or source & Origin $^{a}$ \\
\hline Bordetella pertussis & ATCC 9797 & ATCC \\
\hline Bordetella parapertussis & ATCC 15311 & ATCC \\
\hline Bordetella bronchiseptica & ATCC 19395 & ATCC \\
\hline Bordetella holmesii & ATCC 51541 & ATCC \\
\hline Bordetella avium & LMG 1851 & UZA \\
\hline Bordetella hinzii & LMG 1872 & UZA \\
\hline Bordetella trematum & LMG 5894 & UZA \\
\hline Mycoplasma pneumoniae type 1 & ATCC 29085 (PI 1428) & ATCC \\
\hline Mycoplasma pneumoniae type 2 & ATCC 15492 (MAC) & ATCC \\
\hline Ureaplasma urealyticum & Clinical isolate & UZA \\
\hline Legionella pneumophila & ATCC 33152 & ATCC \\
\hline Chlamydia pneumoniae & TW-183 & \\
\hline Moraxella catarrhalis & ATCC 25238 & ATCC \\
\hline Haemophilus influenzae & ATCC 43065 & ATCC \\
\hline Streptococcus pneumoniae & ATCC 49150 & ATCC \\
\hline Streptococcus pyogenes & ATCC 12344 & ATCC \\
\hline Enterococcus faecalis & ATCC 12984 & ATCC \\
\hline Staphylococcus aureus & ATCC 12600 & ATCC \\
\hline Klebsiella pneumoniae & ATCC 13883 & ATCC \\
\hline Escherichia coli & ATCC 11775 & ATCC \\
\hline Neisseria meningitidis & ATCC 13090 & ATCC \\
\hline Pseudomonas aeruginosa & ATCC 10145 & ATCC \\
\hline Enterobacter aerogenes & ATCC 13048 & ATCC \\
\hline
\end{tabular}

${ }^{a}$ ATCC, American Type Culture Collection; UZA, Universitair Ziekenhuis Antwerpen.

tional PCR assay has been described that fulfills all these recommendations (5).

Here, we describe the development and application of a real-time PCR format using molecular beacons to detect and distinguish between $B$. pertussis and $B$. parapertussis in a test format including an internal control which complies with the consensus recommendations.

\section{MATERIALS AND METHODS}

Bacterial strains. The bacterial strains used to test the specificity of the real-time PCR are presented in Table 1 . All bacteria used for specificity testing were cultured by standard methods. In addition a further 10 culture-positive isolates of $B$. pertussis, 5 culture-positive isolates of $B$. parapertussis, and two B. holmesii PCR-positive samples were selected from our repository and included in the analyses. The $B$. holmesii clinical samples were detected using the PCR described by van der Zee et al. (30)

To evaluate the sensitivity of the PCR test, $B$. pertussis, $B$. parapertussis, and $B$. holmesii were suspended in sterile physiological saline at a concentration equivalent to $10^{8}$ cells per $\mathrm{ml}$, based on McFarland turbidimetric standards. Serial dilutions were made from the suspension, and aliquots were extracted and tested in the PCR to determine sensitivity.

Collection and processing of samples. From April 2001 to December 2001 specimens were obtained from 57 patients who were investigated for B. pertussis infection. Patients presented with cough or wheezing. Nasal swabs (12 samples), throat swabs (37 samples), sputum (3 samples), and nasopharyngeal aspirates (NPAs) (5 samples) were received. Swabs from patients were obtained either in charcoal-based Stuart's transport medium with a Dacron swab, in transport medium, or as dry swabs. The swab used for the transport medium and the dry swab was a cotton-tipped swab, and these samples were used for PCR only. The buffered transport medium contained $0.0185 \% \mathrm{CaCl}_{2}, 0.8 \% \mathrm{NaCl}, 0.04 \% \mathrm{KCl}$, $0.02 \% \mathrm{MgSO}_{4}, 0.0075 \% \mathrm{Na}_{2} \mathrm{HPO}_{4}, 0.1 \%$ glucose, $0.5 \%$ albumin, $0.001 \%$ phenol red, $0.1 \%$ yeast extract, $0.5 \%$ gelatin, and $0.0025 \%$ vancomycin. NPAs and sputum samples were mixed well by vortexing for $30 \mathrm{~s}$, and aliquots were frozen at $-70^{\circ} \mathrm{C}$. Swabs were placed in $0.5 \mathrm{ml}$ of $0.9 \% \mathrm{NaCl}$ prepared in RNase- and DNase-free water, swirled vigorously, and wrung out, and the swab was removed from the specimen before freezing. All specimens were stored at $-70^{\circ} \mathrm{C}$ prior to nucleic acid isolation. All 57 patients included had samples suitable for culture and PCR. Retrospectively, clinical data for these 57 patients were collected. For some cases serological data (2) were obtained by an in-house assay using pertussis toxin or a sonicated whole bacterium as antigen available from the $\mathrm{Na}$ tional Institute of Public Health (Bilthoven, The Netherlands). As a negativecontrol group throat swabs were collected from 20 children with mild respiratory symptoms but with no presence of cough or fever.

Bacterial culture. All specimens suitable for culture were inoculated within $24 \mathrm{~h}$ of collection onto Regan-Lowe medium without antibiotics and ReganLowe medium with cephalexin. Plates were incubated at $35^{\circ} \mathrm{C}$ in a moist Perspex chamber containing air with adequate humidity for up to 7 days. Plates were examined daily for suspect colonies which were identified phenotypically. Suspect colonies for $B$. pertussis and B. parapertussis or B. holmesii were further investigated with agglutinating serum (Murex, Dartford, England).

DNA extraction. Nucleic acids were extracted from bacterial suspensions and clinical specimens with the QiaAmp DNA blood minikit (Qiagen, Hilden, Germany). All samples were extracted according to the manufacturer's instructions with $200 \mu \mathrm{l}$ of samples in each extraction. Negative no-template controls were included in each run and after every 20 samples. In the control sterile distilled water was added instead of specimen.

Primers and probe for conventional PCR. PCR amplification for $B$. pertussis was performed using primers which amplified a 181-bp region of IS481 (accession no. L26973). PCR amplification for B. parapertussis was performed using primers that amplified a 153-bp region of the IS1001 (accession no. X66858). Primers and probe sequences are shown in Table 2.

Conventional PCR. Briefly, the PCR was performed in $50 \mu \mathrm{l}$ of reaction mixture consisting of $25 \mu \mathrm{l}$ of Taq PCR Master Mix (Qiagen), $1.5 \mathrm{mM} \mathrm{MgCl}_{2}, 0.2$ $\mu \mathrm{M}$ (each) primer, and $20 \mu \mathrm{l}$ of extracted DNA. The PCR thermal profile consisted of an initial incubation of $3 \mathrm{~min}$ at $94^{\circ} \mathrm{C}$; followed by 40 cycles of $15 \mathrm{~s}$ at $94^{\circ} \mathrm{C}, 20 \mathrm{~s}$ at $60^{\circ} \mathrm{C}$, and $30 \mathrm{~s}$ at $72^{\circ} \mathrm{C}$; and finally a 10 -min hold at $72^{\circ} \mathrm{C}$. The $B$. pertussis product was captured in an avidin-coated enzyme-linked immunosorbent assay plate and subsequently detected by enzymatic reaction with a probe specific to the IS481 product (34). The probe was labeled with digoxigenin. Inhibition was determined by spiking samples with a $B$. pertussis DNA. The $B$. parapertussis product was detected by agarose gel electrophoresis. Inhibition was determined by spiking duplicate PCRs with B. pertussis or B. parapertussis DNA.

Sequencing of IS1001 for B. parapertussis and B. holmesii. A PCR product was obtained for both $B$. parapertussis and $B$. holmesii by using the primers and PCR as previously described by van der Zee et al. (30). These primers detect both $B$. parapertussis and $B$. holmesii. Nucleotide sequence analysis was set up in $20-\mu \mathrm{l}$ reaction volumes with a BigDye Terminator Cycle Sequencing Ready Reaction kit (Applied Biosystems, Warrington, United Kingdom) according to the manufacture's specifications. Briefly, the reaction mixture contained $3.0 \mathrm{pmol}$ of either PCR primer and purified template DNA $(1 \mu l$ is approximately equal to 200 to 500 ng of DNA). After cycle sequencing the products were purified using an isopropanol precipitation and subsequently analyzed on the ABI 310 automated DNA sequencer (Applied Biosystems). Analysis of the sequences was performed using Vector NTI (Informax).

Primers and probes for real-time PCR. Primer and molecular beacon sequences were selected from the sequence of $B$. pertussis IS481 (accession no. L26973) by using criteria required for design of molecular beacon assays. This region is $99 \%$ homologous to the same region in B. holmesii (accession no. AF349431). Primer and molecular beacon sequences were selected from sequence $B$. parapertussis IS1001 (accession no. X66858) and from the sequencing data for the IS1001 region of both $B$. holmesii and $B$. parapertussis. The real-time PCR was designed so that both $B$. holmesii and B. parapertussis would be detected by the assay. All PCR primers were designed using the Primer 3 program (http://www-genome.wi.mit.edu/cgi-bin/primer/primer3_www.cgi) to ensure minimal self-complementary and no secondary structures.

The molecular beacon was designed using the Mfold Zuker program (http: //www.bioinfo.rpi.edu/applications/mfold/old/dna/). Additional criteria for a good molecular beacon included a melting temperature of $8^{\circ} \mathrm{C}$ over the melting temperature of the primers and the amplicon being relatively short, $<150 \mathrm{bp}$. The stem sequence was selected to have a melting temperature compatible with the molecular beacon. The beacon formed a stable structure at 50 to $55^{\circ} \mathrm{C}$, the proposed annealing temperature, with no secondary structures. A BLAST search was performed to check the specificity of the DNA sequences of the primers and probes. For $B$. pertussis the fluorescent reporter on the $5^{\prime}$ end of the probe was 6-carboxyfluorescein and the quencher on the $3^{\prime}$ end was Dabcyl. For B. parapertussis the fluorescent reporter on the $5^{\prime}$ end of the probe was Texas Red and the quencher on the $3^{\prime}$ end was Black Hole Quencher 2. The molecular beacon and primers were prepared by Biolegio (Malden, The Netherlands). Selected primers and probe are shown in Table 2.

Real-time PCR. Real-time PCR for B. pertussis was performed in $50 \mu \mathrm{l}$ of reaction mixture consisting of $25 \mu$ l of Hotstar Taq Master Mix (Qiagen), $3.5 \mathrm{mM}$ 
TABLE 2. Primers and probes for PCR

\begin{tabular}{|c|c|c|c|}
\hline Assay format and target & Primer or probe sequence ${ }^{a}$ & Primer or probe & Amplicon size (bp) \\
\hline \multicolumn{4}{|l|}{ PCR } \\
\hline \multirow[t]{3}{*}{ IS481 } & GATTCAATAGGTTGTATGCATGGTT & Forward primer & \multirow[t]{3}{*}{181} \\
\hline & TTCAGGCACACAAACTTGATGGGCG & Reverse primer & \\
\hline & CGTCGACTCGAAATGGTCCAGC & Probe & \\
\hline \multirow[t]{2}{*}{ IS1001 } & CGCTGGCTGCTGCTGCGCAA & Forward primer & \multirow[t]{2}{*}{171} \\
\hline & GTGGTTCCACGCTTGTCTTG & Reverse primer & \\
\hline \multicolumn{4}{|l|}{ RT-PCR ${ }^{b}$} \\
\hline \multirow[t]{3}{*}{ IS481 } & TCAATAGGTTGTATGCATGG & Forward primer & \multirow[t]{3}{*}{154} \\
\hline & GATCAATTGCTGGACCATT & Reverse primer & \\
\hline & FAM-GCAGGCGGCCGGATGAACACCCATAAGCCTGC-Dabcyl & Molecular beacon & \\
\hline \multirow[t]{3}{*}{ IS1001 } & CCATGTCGTGGCCAAGTA & Forward primer & \multirow[t]{3}{*}{186} \\
\hline & TGGTTGGCTTGCAGCAA & Reverse primer & \\
\hline & Texas red-GCAGGCGCTGGCTRCTGCTGCGCAAGCCTGC-BHQ2 & Molecular beacon & \\
\hline \multirow[t]{3}{*}{ PhHV gB } & ATGCATTTAAAACCCTCAAA & Forward primer & \multirow[t]{3}{*}{140} \\
\hline & GCATCAACTTCTTCGACAAT & Reverse primer & \\
\hline & Су-5-GTCGCCCCTGGTTTTTATCGTACGGGAACAGGCGAC-BHQ2 & Molecular beacon & \\
\hline
\end{tabular}

\footnotetext{
${ }^{a}$ Underlined nucleotides indicate the stem structure of the molecular beacon.

${ }^{b}$ RT-PCR, real-time PCR.
}

$\mathrm{MgCl}_{2}, 0.1 \mu \mathrm{M}$ (each) primer, $0.34 \mu \mathrm{M}$ molecular beacon, and $10 \mu \mathrm{l}$ of template For B. parapertussis the real-time PCR was performed in $50 \mu \mathrm{l}$ of reaction mixture consisting of Hotstar Taq Master Mix, $5.5 \mathrm{mM} \mathrm{MgCl}_{2}, 0.2 \mu \mathrm{M}$ (each) primer, $0.45 \mu \mathrm{M}$ molecular beacon, and $10 \mu \mathrm{l}$ of template The PCR thermal profile consisted of an initial incubation of $15 \mathrm{~min}$ at $95^{\circ} \mathrm{C}$ followed by 50 cycles of $30 \mathrm{~s}$ at $95^{\circ} \mathrm{C}, 30 \mathrm{~s}$ at $55^{\circ} \mathrm{C}$, and $30 \mathrm{~s}$ at $72^{\circ} \mathrm{C}$. Amplification, detection, and data analysis were performed with an iCycler IQ Real-Time Detection system (BioRad, Veenendaal, The Netherlands).

Internal control real-time PCR. The quality of nucleic acid extraction as well as inhibition of the real-time PCR was monitored by amplification of a phocine herpesvirus (PhHV) spike. This method has been described by Niesters using Taqman probes (19). For implementation in the B. pertussis real-time PCR the assay was redesigned using primer and molecular beacon as a probe, with the molecular beacon labeled with Cy-5 (28). The PhHV-optimized primer and probe concentrations were used in the $B$. pertussis real-time PCR. Each sample was spiked with a dilution of viral stock of $\mathrm{PhHV}$ which was coextracted with the sample, and the assay was performed as a duplex PCR.

Inter- and intra-assay variability. DNA was extracted from $B$. pertussis ATCC 9797 and stored in AE buffer. The DNA was diluted to a concentration equivalent to $100 \mathrm{CFU} / \mathrm{ml}$ and stored in small aliquots at $-20^{\circ} \mathrm{C}$. In order to determine inter- and intra-assay variation an aliquot was thawed and run in quintuplicate in five consecutive runs of the multiplex real-time PCR assay.

Analysis of results. All clinical data and laboratory results were coded and entered into a database. A pertussis case was defined as cough lasting at least 2 weeks, paroxysms of coughing or vomiting, and one or more of the following symptoms or characteristics: apnea or cyanosis, subconjunctival bleeding, lymphocytosis, or a recent contact (up to 3 weeks) with a whooping cough patient. In addition to these clinical criteria whooping cough was also considered in the case of (i) positive culture of $B$. pertussis or (ii) serological confirmation according to the criteria of the National Institute of Public Health (2).

The clinical sensitivities and specificities and predictive values of the results were determined in two frequency tables with the clinical criteria for pertussis as the gold standard.

\section{RESULTS}

Real-time PCR for $B$. pertussis. The real-time PCR for $B$. pertussis was optimized first as a monoplex assay with a 10 -fold dilution series of both $B$. pertussis DNA and B. holmesii DNA. PCR efficiency with the iCycler software was 98 and 95\% for B. pertussis and B. holmesii, respectively. The monoplex real-time PCR was thereafter compared to duplex PCR with the PhHV internal control. The threshold cycle $(\mathrm{Ct})$ values obtained in the monoplex assay and the duplex assay showed a mean difference of values of 0.3 . In addition the clinical sensitivity of the assay was not affected by duplexing.

Sequence analysis of IS1001 for B. holmesii and B. parapertussis. A 440-bp product amplified from the IS1001 fragment of both $B$. holmesii and B. parapertussis was sequenced. The resulting sequences were aligned. Based on the consensus regions a real-time PCR for both targets was designed. There was approximately $87 \%$ homology between the two Bordetella spp. The sequence data were compared to sequence data from the whole IS1001 for both B. parapertussis and the equivalent region for $B$. holmesii, and there was good correlation between the sequencing results. From the sequence data it appears that this sequence is related to an insertion sequence for $B$. holmesii.

Real-time PCR for $\boldsymbol{B}$. parapertussis. The real-time PCR for B. parapertussis was optimized as a monoplex assay in the same way as the $B$. pertussis assay. PCR efficiency with the iCycler software was 102 and $105 \%$ for B. parapertussis and B. holmesii, respectively. Both assays had the same PCR protocol, so they could be performed in a single plate.

Specificity. Primers and probes of the $B$. pertussis real-time PCR assay successfully amplified DNA from both $B$. pertussis and $B$. holmesii and none of the other Bordetella species or other respiratory bacteria listed in Table 1 . Additionally 10 positive isolates for $B$. pertussis and two clinical samples positive for $B$. holmesii were detected by the real-time PCR assay. The primers and probes from the $B$. parapertussis real-time PCR assay successfully amplified DNA from $B$. parapertussis and $B$. holmesii and none of the other Bordetella species or other respiratory bacteria listed in Table 1 . Additionally, the five $B$. parapertussis-positive isolates and two clinical samples positive for $B$. holmesii were detected by the real-time PCR assay. Application of the results to diagnose infection by $B$. pertussis and B. parapertussis is summarized in Table 3. 
TABLE 3. Use of assays to determine diagnosis

\begin{tabular}{llcc}
\hline \multirow{2}{*}{ Diagnosis } & \multicolumn{3}{c}{ Result by RT-PCR ${ }^{a}$} \\
\cline { 2 - 4 } & $\begin{array}{c}\text { B. per- } \\
\text { tussis }\end{array}$ & $\begin{array}{l}\text { B. para- } \\
\text { pertussis }\end{array}$ & $\begin{array}{c}\text { Internal } \\
\text { control }\end{array}$ \\
\hline B. pertussis & $\mathrm{Pos}$ & $\mathrm{Neg}$ & $\mathrm{Pos}$ \\
$\begin{array}{l}\text { B. holmesii } \text { or coinfection } \\
\text { B. parapertussis }\end{array}$ & $\mathrm{Pos}$ & $\mathrm{Pos}$ & $\mathrm{Pos}$ \\
$\begin{array}{l}\text { Valid result, negative for } \text { B. pertussis, } \\
\text { B. parapertussis, and } B \text {. holmesii }\end{array}$ & $\mathrm{Neg}$ & $\mathrm{Pos}$ & $\mathrm{Pos}$ \\
$\begin{array}{l}\text { Inhibition or invalid extraction- } \\
\text { test needs to be repeated }\end{array}$ & $\mathrm{Neg}$ & $\mathrm{Neg}$ & $\mathrm{Pos}$ \\
\hline
\end{tabular}

${ }^{a}$ RT-PCR, real-time PCR; Pos, positive; Neg, negative.

${ }^{b}$ Differentiation by Sequencing of real-time PCR product

Sensitivity. DNA was extracted from dilution series of the $B$. pertussis suspensions and subjected to PCR amplification. A sample was interpreted as positive if the relative fluorescence crossed the threshold as determined by the iCycler detection system software. The sensitivity of the real-time PCR assays was shown to be 1 to $10 \mathrm{CFU} / \mathrm{ml}$, which was similar to that of the conventional PCR. B. holmesii could be detected with a sensitivity of $10 \mathrm{CFU} / \mathrm{ml}$. The sensitivity of the B. parapertussis real-time PCR assay, applied to a dilution series of $B$. parapertussis and $B$. holmesii suspension, was determined to be 10 $\mathrm{CFU} / \mathrm{ml}$ for both targets.

Evaluation of PCR inhibition. For application of PCR to clinical specimens in a diagnostic laboratory sufficient controls should be included. Besides specific positive and negative PCR controls, DNA isolation and inhibition should be monitored as well. In this real-time assay the PhHV was used as an internal control.

A dilution series of $\mathrm{PhHV}$ from $10^{8}$ to $10^{1} \mathrm{PFU}$ of $\mathrm{PhHV} / \mathrm{ml}$ was coamplified with the $B$. pertussis targets, which did not significantly affect the efficiency of the reaction. In addition, a spike of $10^{3} \mathrm{PFU}$ of $\mathrm{PhHV} / \mathrm{ml}$ in the sample prior to extraction resulted in a positive result at a $\mathrm{Ct}$ value of 33 to 34 , and this was not significantly affected by amplification of a dilution series of $B$. pertussis and resulted in the same clinical sensitivity. Thus, spiking and coamplification of $\mathrm{PhHV}$ and the Bordetella targets resulted in similar $\mathrm{Ct}$ values in comparison to amplification without spikes (Table 4).

Reproducibility of $\boldsymbol{B}$. pertussis real-time PCR assay. Samples containing $100 \mathrm{CFU} / \mathrm{ml}$ were assayed in quintuplicate in the multiplex assay to determine the interassay and intra-assay variability. As determined from the $\mathrm{Ct}$ values obtained from five consecutive runs the interrun variation was 0.7 for this standard sample. The mean of the intra-assay variability was 0.36 (range, 0.2 to 0.6 ).

TABLE 4. Ct values for B. pertussis CFU-per-milliliter dilution series with and without the PhHV spike

\begin{tabular}{lcc}
\hline \multirow{2}{*}{ Dilution $(\mathrm{CFU} / \mathrm{ml})$} & \multicolumn{2}{c}{ Ct value } \\
\cline { 2 - 3 } & No spike & With spike \\
\hline 10,000 & 27.5 & 28 \\
1,000 & 32.1 & 33 \\
100 & 35.9 & 36.2 \\
10 & 39 & 40.2 \\
1 & 45.5 & 46.7 \\
\hline
\end{tabular}

TABLE 5. Results of culture, conventional PCR, and real-time PCR from 57 patient samples with clinically suspected pertussis infection ${ }^{a}$

\begin{tabular}{|c|c|c|c|c|c|}
\hline \multirow[t]{2}{*}{ Method } & \multicolumn{3}{|c|}{ No. (\%) positive } & \multicolumn{2}{|c|}{$\begin{array}{l}\text { No. with } \\
\text { result in } \\
\text { clinical per- } \\
\text { tussis test }\end{array}$} \\
\hline & B. pertussis & B. parapertussis & B. holmesii & Pos & Neg \\
\hline Culture & $8(14)$ & 0 & 0 & 8 & 13 \\
\hline Conv. PCR & $18(33)$ & 0 & NT & 17 & 3 \\
\hline RT-PCR & $22(39)$ & 0 & 0 & 21 & 0 \\
\hline Total & 57 & 57 & 57 & 21 & \\
\hline
\end{tabular}

${ }^{a}$ Abbreviations: Pos, positive; Neg, negative; Conv. PCR, conventional PCR; RT-PCR, real-time PCR; NT, not tested.

Clinical evaluation of real-time PCRs. During the 8-month period described, 57 patients were seen and clinically suspected of Bordetella infection. Twenty-one of these 57 fulfilled the clinical definition for pertussis infection. Real-time PCR, conventional PCR, and culture were performed on clinical samples of these patients. Eight of the 57 (14\%) were found positive by culture, 19 of 57 were found positive by conventional PCR (33\%), and 22 of $57(39 \%)$ were found positive by real-time PCR (Table 5). One sample was inhibitory, as the PhHV spike was not amplified. This sample was also inhibitory by conventional PCR and was culture negative (serology was not available). When the $B$. pertussis assays were compared with the clinical standard for $B$. pertussis infection, the sensitivity was 38,85 , and $100 \%$; the specificity was 100,97 , and 97\%; the positive predictive value was 100, 93, and 95\%; and the negative predictive value was 73,92 , and $100 \%$ for culture, conventional PCR, and real-time PCR, respectively. As shown, there were three samples positive by real-time PCR only, and all had clinical criteria for disease (Fig. 1). One of the 11 samples positive by both $B$. pertussis PCR assays did not conform to clinical disease criteria (Fig. 1). Individual sensitivities

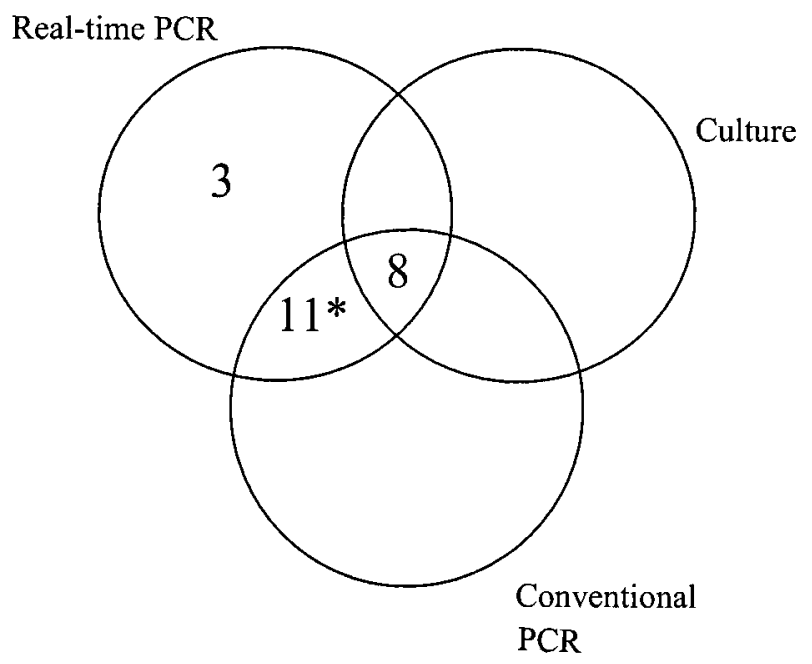

FIG. 1. Venn diagram showing assays demonstrating B. pertussis positivity. One sample positive by both PCR assays (*) did not conform to the clinical definition of pertussis. 
and specificities for different samples were assessed for culture and real-time PCR. The sensitivity for culture in throat swab, nasal swab, sputum, and NPAs was 18, 16, 100, and 100\%, respectively, and the specificity for all samples was $100 \%$. The sensitivity for real-time PCR was $100 \%$ for all samples, and the specificity was $100 \%$ for all samples except throat swabs, which had a specificity of $97 \%$. The mean $\mathrm{Ct}$ value for the patients with a positive culture was 28.8 (range, 17.1 to 34.7 ), and for patients with no positive culture the mean was 40.3 (range, 33.3 to 46 ). None of the 57 patients were positive for either $B$. parapertussis or $B$. holmesii by any of the methods.

\section{DISCUSSION}

The real-time PCR assay described provides an effective way to detect and discriminate between $B$. pertussis and $B$ parapertussis infection. PCR has repeatedly been shown to be more sensitive than culture and direct fluorescent-antibody assay for detection of $B$. pertussis $(7,13,23,29,30,33)$. However, analysis of discrepant results and assessment of clinical performance of PCR have been carried out less frequently $(13,33)$. The real-time PCR assays have excellent analytical sensitivity as shown previously for other PCR methods $(11,13,26)$. PCR is much more sensitive than culture because culture requires viable microorganisms. B. pertussis is a particularly labile organism, and most diagnostic laboratories are unable to culture the patient's material directly from the patient. In this study culture sensitivity was $38 \%$, but others have reported rates of $15.2 \%$ (13) and $73.4 \%$ (33). The difference in sensitivity rates can be explained by differences in transportation to the laboratory, differences in when the sample is inoculated onto agar, and also the age of the patients. For example, Wadowsky et al., who achieved a culture sensitivity of $73 \%$, immediately inoculated the samples onto agar after collection and tested a mainly pediatric population (33). A comparison of sample types showed that NPAs and sputum were better for culture, but the numbers were small, so conclusions are limited. In this study it appears that the real-time PCR has greater sensitivity than conventional PCR, as three clinical samples were positive in the real-time PCR only. Inclusion of the molecular beacon in the real-time assay may create a more stable template for the PCR, hence providing improved efficiency and thus sensitivity. In addition, owing to the software analysis with real-time PCR, which calculates efficiency, a more optimal reaction is achieved with the real-time assay than with conventional PCR assays. This also improves the sensitivity of the real-time PCR. Samples with lower $\mathrm{Ct}$ values in the real-time PCR assay were more likely to be culture positive and thus have a higher concentration of bacteria. The mean $\mathrm{Ct}$ in culture-positive samples was 28.8 compared with a mean $\mathrm{Ct}$ of 40 in culture-negative samples, providing further evidence that the PCR assay had increased sensitivity to culture.

In this study the real-time PCR assays were performed in two tubes, although a multiplex format detecting all three Bordetella targets and the internal control in a single tube was possible with similar sensitivities for $B$. pertussis and $B$. parapertussis (results not shown). The sensitivity of $B$. holmesii was 3 logs less in the multiplex format than in the two-well format. As the B. holmesii DNA had to be detected by both molecular beacons, this may have affected the efficiency of the multiplex format.

A PhHV spike was added to the clinical samples prior to DNA isolation and coamplified in the B. pertussis real-time PCR assay. In this way, control of the DNA isolation procedure as well as a check for inhibition was achieved. The level of inhibition was low, as only 1 in 57 samples (2\%) was inhibited. Hence, the use of the extraction with guanidine thiocyanate to lyse cells, followed by washing and precipitation of nucleic acids, is very effective at removing PCR inhibition, although when sputum samples are used more inhibition can be expected (9). In this study, swabs were the main samples collected, and other studies using only dry swabs have also found very low rates of inhibition (13).

The positive predictive value of culture and B. pertussis realtime PCR was 100 and 95\%, respectively, using the clinical criteria as the gold standard. This value is very similar to that of Loeffelholz et al. (13). The one PCR-positive patient not meeting the criteria did have a paroxysmal cough. However, serology was not performed and $B$. pertussis data for pertussis in the local environment were not available, so it may be that this patient actually had $B$. pertussis infection but that the information to assess by clinical criteria was unavailable.

When PCR is used in the diagnostic laboratory, concerns remain regarding false-positive results due to contamination or cross-reactivity. The use of real-time PCR helps to minimize the risk of false positives, as there is no need to handle amplified material. In this study all samples were also tested by a second conventional PCR, which, although targeting the same repeat region, had different primers and probe, and the sequences did not overlap. Nineteen of the 22 positives were confirmed by this method. The other three all met clinical criteria for disease. Confirmation of positives, especially in an outbreak situation, by a second PCR is recommended (12). In addition, consensus recommendations require the use of an alternative PCR to confirm questionable results (17). In the present study all samples were run with negative controls and all sample processing and testing were performed according to the regulations in the laboratory, which prevent PCR contamination. None of the negative controls were positive, and also each sample with a positive signal was repeatedly positive.

The clinical significance of detecting $B$. holmesii is not yet fully understood, but the bacterium has been isolated from the respiratory tract $(16,35)$ and been reported to cause disease (24). However, because of the design of the PCR assays described it is possible to provide a presumptive diagnosis for $B$. holmesii, as both assays detect $B$. holmesii. However, a mixed infection of $B$. pertussis and $B$. parapertussis is also possible but rare, and to date only one has been reported (11). Essentially any PCR assay targeting the IS481 region will detect $B$. pertussis and B. holmesii (22), so the use of the B. parapertussis PCR, which detects both $B$. holmesii and $B$. parapertussis, enables $B$. holmesii infection or a dual infection of $B$. pertussis and $B$. parapertussis to be diagnosed. In the rare event that this occurs, the PCR product from the $B$. parapertussis PCR can be sequenced and $B$. holmesii infection can be distinguished from a dual infection. Of other Bordetella species such as B. bronchiseptica, B. avium, B. trematum, and B. hinzii, it is only $B$. bronchiseptica that has been reported to cause respiratory infection in immunocompromised hosts (14). So the possibility 
of this pathogen in immunocompromised patients should be considered separately.

Molecular methods have been shown to be highly sensitive and specific for $B$. pertussis, but one standardized method has yet to be adopted, although consensus recommendations have been published (17). Only the assay published by Farrell et al. complies with these recommendations, except that it does not distinguish between B. parapertussis and B. bronchiseptica (5). The assay described here complies with all the recommendations, except that the confirmatory PCR, although it uses different primers, targets the same IS481 sequence.

The real-time PCR described here for B. pertussis and $B$. parapertussis provides sensitive and specific diagnosis of $B$. pertussis and $B$. parapertussis infections. The protocol used was able to complete analysis of 30 samples including controls in 4 to $5 \mathrm{~h}$, including extraction and assay time, and therefore is suitable for implementation in the diagnostic laboratory.

\section{ACKNOWLEDGMENTS}

This study was supported by European Commission (Framework V) grant no. QLK2-CT-2000-00294.

We thank Bert Niesters, Erasmus Medical Center, Rotterdam, The Netherlands, for providing the PhHV and Katherine Loens, University Hospital, Antwerp, Belgium, for some of the Bordetella strains.

\section{REFERENCES}

1. Cherry, J. D. 1996. Historical review of pertussis and the classical vaccine. J. Infect. Dis. 174(Suppl. 3):S259-S263.

2. de Melker, H. E., F. G. Versteegh, M. A. Conyn-Van Spaendonck, L. H. Elvers, G. A. Berbers, A. van Der Zee, and J. F. Schellekens. 2000. Specificity and sensitivity of high levels of immunoglobulin $\mathrm{G}$ antibodies against pertussis toxin in a single serum sample for diagnosis of infection with Bordetella pertussis. J. Clin. Microbiol. 38:800-806.

3. Douglas, E., J. G. Coote, R. Parton, and W. McPheat. 1993. Identification of Bordetella pertussis in nasopharyngeal swabs by PCR amplification of a region of the adenylate cyclase gene. J. Med. Microbiol. 38:140-144.

4. Farrell, D. J., G. Daggard, and T. K. Mukkur. 1999. Nested duplex PCR to detect Bordetella pertussis and Bordetella parapertussis and its application in diagnosis of pertussis in nonmetropolitan Southeast Queensland, Australia J. Clin. Microbiol. 37:606-610.

5. Farrell, D. J., M. McKeon, G. Daggard, M. J. Loeffelholz, C. J. Thompson, and T. K. Mukkur. 2000. Rapid-cycle PCR method to detect Bordetella pertussis that fulfills all consensus recommendations for use of PCR in diagnosis of pertussis. J. Clin. Microbiol. 38:4499-4502.

6. Furuya, D., A. Yagihashi, T. Endoh, N. Uehara, N. Fujii, S. Chiba, and N. Watanabe. 1999. Simultaneous amplification of Bordetella repeated insertion sequences and toxin promoter region gene by polymerase chain reaction. Immunopharmacol. Immunotoxicol. 21:55-63.

7. He, Q., M. K. Viljanen, H. Arvilommi, B. Aittanen, and J. Mertsola. 1998 Whooping cough caused by Bordetella pertussis and Bordetella parapertussis in an immunized population. JAMA 280:635-637.

8. Heininger, U., K. Stehr, S. Schmitt-Grohe, C. Lorenz, R. Rost, P. D. Christenson, M. Uberall, and J. D. Cherry. 1994. Clinical characteristics of illness caused by Bordetella parapertussis compared with illness caused by Bordetella pertussis. Pediatr. Infect. Dis. J. 13:306-309.

9. Ieven, M., D. Ursi, H. Van Bever, W. Quint, H. G. Niesters, and H. Goossens. 1996. Detection of Mycoplasma pneumoniae by two polymerase chain reactions and role of $M$. pneumoniae in acute respiratory tract infections in pediatric patients. J. Infect. Dis. 173:1445-1452.

10. Khetsuriani, N., K. Bisgard, D. R. Prevots, M. Brennan, M. Wharton, S. Pandya, A. Poppe, K. Flora, G. Dameron, and P. Quinlisk. 2001. Pertussis outbreak in an elementary school with high vaccination coverage. Pediatr. Infect. Dis. J. 20:1108-1112.

11. Kosters, K., U. Reischl, J. Schmetz, M. Riffelmann, and C. H. Wirsing von Konig. 2002. Real-time LightCycler PCR for detection and discrimination of Bordetella pertussis and Bordetella parapertussis. J. Clin. Microbiol. 40:17191722.

12. Lievano, F. A., M. A. Reynolds, A. L. Waring, J. Ackelsberg, K. M. Bisgard, G. N. Sanden, D. Guris, A. Golaz, D. J. Bopp, R. J. Limberger, and P. F. Smith. 2002. Issues associated with and recommendations for using PCR to detect outbreaks of pertussis. J. Clin. Microbiol. 40:2801-2805.

13. Loeffelholz, M. J., C. J. Thompson, K. S. Long, and M. J. Gilchrist. 1999. Comparison of PCR, culture, and direct fluorescent-antibody testing fo detection of Bordetella pertussis. J. Clin. Microbiol. 37:2872-2876.
14. Lorenzo-Pajuelo, B., J. L. Villanueva, J. Rodriguez-Cuesta, N. VergaraIrigaray, M. Bernabeu-Wittel, A. Garcia-Curiel, and G. Martinez de Tejada. 2002. Cavitary pneumonia in an AIDS patient caused by an unusual Bordetella bronchiseptica variant producing reduced amounts of pertactin an other major antigens. J. Clin. Microbiol. 40:3146-3154.

15. Matthews, R. C., N. Golbang, W. M. Bruck, D. Owen, A. Bailey, V. Weston, and J. R. Kerr. 1999. Semiquantitative polymerase chain reaction enzyme immunoassay for the diagnosis of pertussis. Eur. J. Clin. Microbiol. Infect. Dis. 18:748-750.

16. Mazengia, E., E. A. Silva, J. A. Peppe, R. Timperi, and H. George. 2000. Recovery of Bordetella holmesii from patients with pertussis-like symptoms: use of pulsed-field gel electrophoresis to characterize circulating strains. J. Clin. Microbiol. 38:2330-2333.

17. Meade, B. D., and A. Bollen. 1994. Recommendations for use of the polymerase chain reaction in the diagnosis of Bordetella pertussis infections. J. Med. Microbiol. 41:51-55.

18. Muller, F. M., U. Heininger, N. Schnitzler, P. Kockelkorn, O. Cloot, C. Lorenz, and G. Hase. 1994. Discrimination of Bordetella parapertussis and Bordetella pertussis organisms from clinical isolates by PCR using biotinlabelled oligonucleotide probes. Mol. Cell. Probes 12:213-217.

19. Niesters, H. G. 2001. Quantification of viral load using real-time amplification techniques. Methods 25:419-429.

20. Nygren, M., E. Reizenstein, M. Ronaghi, and J. Lundeberg. 2000. Polymorphism in the pertussis toxin promoter region affecting the DNA-based diagnosis of Bordetella infection. J. Clin. Microbiol. 38:55-60.

21. Poddar, S. K., and C. T. Le. 2001. Bordetella pertussis detection by spectrofluorometry using polymerase chain reaction (PCR) and a molecular beacon probe. Mol. Cell. Probes 15:161-167.

22. Reischl, U., N. Lehn, G. N. Sanden, and M. J. Loeffelholz. 2001. Real-time PCR assay targeting IS481 of Bordetella pertussis and molecular basis for detecting Bordetella holmesii. J. Clin. Microbiol. 39:1963-1966.

23. Reizenstein, E., L. Lindberg, R. Mollby, and H. O. Hallander. 1996. Validation of nested Bordetella PCR in pertussis vaccine trial. J. Clin. Microbiol. 34:810-815.

24. Russell, F. M., J. M. Davis, M. J. Whipp, P. H. Janssen, P. B. Ward, J. R. Vyas, M. Starr, S. M. Sawyer, and N. Curtis. 2001. Severe Bordetella holmesii infection in a previously healthy adolescent confirmed by gene sequence analysis. Clin. Infect. Dis. 33:129-130.

25. Qin, X., D. K. Turgeon, B. P. Ingersoll, P. W. Monsaas, C. J. Lemoine, T. Tsosie, L. O Stapp, and P. M. Abe. 2002. Bordetella pertussis PCR: simultaneous targeting of signature sequences. Diagn. Microbiol. Infect. Dis. 43: $269-275$.

26. Sloan, L. M., M. K. Hopkins, P. S. Mitchell, E. A. Vetter, J. E. Rosenblatt, W. S. Harmsen, F. R. Cockerill, and R. Patel. 2002. Multiplex LightCycler PCR assay for detection and differentiation of Bordetella pertussis and Bordetella parapertussis in nasopharyngeal specimens. J. Clin. Microbiol. 40:96100.

27. Stojanov, S., J. Liese, and B. H. Belohradsky. 2000. Hospitalization and complications in children under 2 years of age with Bordetella pertussi. infection. Infection 28:106-110.

28. Templeton, K. E., S. A. Scheltinga, A. W. Graffelman, J. M. van Schie, J. W. Crielaard, P. Sillekens, P. J. van den Broek, H. Goossens, M. F. C. Beersma, and E. C. J. Claas. 2003. Comparison and evaluation of real-time PCR, real-time nucleic acid sequence-based amplification, conventional PCR, and serology for diagnosis of Mycoplasma pneumoniae. J. Clin. Microbiol. 41: 4366-4371.

29. Tilley, P. A., M. V. Kanchana, I. Knight, J. Blondeau, N. Antonishyn, and H. Deneer. 2000. Detection of Bordetella pertussis in a clinical laboratory by culture, polymerase chain reaction, and direct fluorescent antibody staining; accuracy and cost. Diagn. Microbiol. Infect. Dis. 37:17-23.

30. van der Zee, A., C. Agterberg, M. Peeters, J. Schellekens, and F. R. Mooi. 1993. Polymerase chain reaction assay for pertussis: simultaneous detection and discrimination of Bordetella pertussis and Bordetella parapertussis. J. Clin. Microbiol. 31:2134-2140.

31. van der Zee, A., C. Agterberg, M. Peeters, F. Mooi, and J. Schellekens. 1996. A clinical validation of Bordetella pertussis and Bordetella parapertussis polymerase chain reaction: comparison with culture and serology using samples from patients with suspected whooping cough from a highly immunized population. J. Infect. Dis. 174:89-96.

32. van der Zee, A., F. Mooi, J. Van Embden, and J. Musser. 1997. Molecular evolution and host adaptation of Bordetella spp.: phylogenetic analysis using multilocus enzyme electrophoresis and typing with three insertion sequences. J. Bacteriol. 179:6609-6617.

33. Wadowsky, R. M., R. H. Michaels, T. Libert, L. A. Kingsley, and G. D. Ehrlich. 1996. Multiplex PCR-based assay for detection of Bordetella pertussis in nasopharyngeal swab specimens. J. Clin. Microbiol. 34:2645-2649.

34. Wiedbrauk, D. L., and A. M. Drevon. 1995. Nucleic acid detection methods, p. 1-22. In D. L. Wiedbrauk and D. H. Farkas (ed.), Molecular methods for virus detection. Academic Press, Inc., San Diego, Calif.

35. Yih, W. K., E. A. Silva, J. Ida, N. Harrington, S. M. Lett, and H. George. 1999. Bordetella holmesii-like organisms isolated from Massachusetts patients with pertussis-like symptoms. Emerg. Infect. Dis. 5:441-443. 